2009;50:1783-1803). (Respond: Dr Gonzalo Alarcon, Dept Clinical Neurophysiology, King's College Hospital, Denmark Hill, London SE5 8ED, UK. E-mail: gonzalo.alarcon@iop.kcl.ac.uk).

COMMENT. Cortical responses to SPES in children are similar to those observed in adults. Abnormal SPES responses (DRs and RRs) correlate with epileptogenic cortex, and are useful in the presurgical evaluation and positioning of electrodes. The method may be used with or without anesthesia (Valentin et al, King's College Hospital, unpublished observation).

\title{
KETOGENIC DIET AND HORMONAL THERAPY IN PREVENTION OF EVOLUTION OF WEST SYNDROME TO LENNOX-GASTAUT
}

Medical records of 98 patients diagnosed with West syndrome and monitored at Sanggye Paik Hospital, Seoul, Korea, for at least 3 years were retrospectively reviewed to assess etiology, age at onset, value of various therapies, and the rate of evolution from West syndrome to Lennox-Gastaut syndrome. During follow-up, West syndrome evolved to Lennox-Gastaut syndrome in 48 of the 98 (49\%) patients. Etiology of West syndrome was cryptogenic in $36(36.7 \%)$ and symptomatic in 62 (63.3\%). Patients with normal psychological development before seizure onset were excluded. All patients had hypsarrhythmia and infantile spasms. Treatment of West syndrome varied as follows: antiepileptic drugs in 31 patients, ketogenic diet in 33, prednisolone in 45, ACTH in 15, surgery in 3, and herbal medication or no treatment in 4 patients. Age at onset of seizures (mean, $5.8+/-2.4$ mos) or disease etiology was not related to development of LennoxGastaut syndrome. Risk of evolving to Lennox-Gastaut syndrome in patients treated with anticonvulsant drugs was 17 in 31 (55\%). Risk was significantly lower in patients treated with ketogenic diets $(10 / 33,30 \%)$ or hormonal therapy $(26 / 60,43 \%)$ or a combination of both (7/27, 26\%), $(\mathrm{P}<0.05)$. (You SJ, Kim HD, Kang H-C. Factors influencing the evolution of West syndrome to Lennox-Gastaut syndrome. Pediatr Neurol Aug 2009;41:111-113). (Respond: Dr Kang, 134 Shinchon Dong, Seodaemun Gu, Seoul 120-752, Korea. E-mail: hipo0207@yuhs.ac).

COMMENT. The ketogenic diet, prednisolone or ACTH or a combination of both diet and hormonal therapy may prevent the development of encephalopathy and LennoxGastaut syndrome in patients with West syndrome. It is estimated that $20-50 \%$ of West syndrome cases evolve to Lennox-Gastaut syndrome; $70-80 \%$ of Lennox-Gastaut cases have no history of West syndrome. The relatively higher risk of development of Lennox-Gastaut after West syndrome in the present study may be related to the exclusion of West syndrome patients with normal neuropsychological development prior to onset of infantile spasms. That the evolution to Lennox-Gastaut syndrome is significantly reduced by treatment with ketogenic diet, hormonal therapy or both suggests that these therapies may modify the underlying encephalopathic process in West and Lennox-Gastaut syndromes. Age and brain maturation are not the primary factors in the development of Lennox-Gastaut syndrome following West syndrome. 